ARTICLE

Received 4 Aug 2015 | Accepted 22 Oct 2015 | Published 27 Nov 2015

DOI: $10.1038 /$ ncomms 9970

OPEN

\title{
Characterizing entanglement of an artificial atom and a cavity cat state with Bell's inequality
}

Brian Vlastakis ${ }^{1, \star}$, Andrei Petrenko ${ }^{1, \star}$, Nissim Ofek${ }^{1}$, Luyan Sun ${ }^{1}$, Zaki Leghtas ${ }^{1}$, Katrina Sliwa1, Yehan Liu ${ }^{1}$ Michael Hatridge ${ }^{1}$, Jacob Blumoff ${ }^{1}$, Luigi Frunzio', Mazyar Mirrahimi,2, Liang Jiang ${ }^{1}$, M.H. Devoret ${ }^{1}$ \& R.J. Schoelkopf ${ }^{1}$

The Schrodinger's cat thought experiment highlights the counterintuitive concept of entanglement in macroscopically distinguishable systems. The hallmark of entanglement is the detection of strong correlations between systems, most starkly demonstrated by the violation of a Bell inequality. No violation of a Bell inequality has been observed for a system entangled with a superposition of coherent states, known as a cat state. Here we use the ClauserHorne-Shimony-Holt formulation of a Bell test to characterize entanglement between an artificial atom and a cat state, or a Bell-cat. Using superconducting circuits with high-fidelity measurements and real-time feedback, we detect correlations that surpass the classical maximum of the Bell inequality. We investigate the influence of decoherence with states up to 16 photons in size and characterize the system by introducing joint Wigner tomography. Such techniques demonstrate that information stored in superpositions of coherent states can be extracted efficiently, a crucial requirement for quantum computing with resonators.

\footnotetext{
${ }^{1}$ Departments of Physics and Applied Physics, Yale University, New Haven, Connecticut 06510, USA. ${ }^{2}$ INRIA Paris-Rocquencourt, Domaine de Voluceau, B.P. 105, 78153 Le Chesnay Cedex, France. * These authors contributed equally to this work. Correspondence and requests for materials should be addressed to B.V. (email: brian.vlastakis@yale.edu) or to R.J.S. (email: robert.schoelkopf@yale.edu).
} 
uantum information processing necessitates the creation and detection of complex entangled states. Many physical implementations aim to encode quantum information into large registers of entangled two-level systems, or qubits. Although originally proposed to investigate local hidden variable theory $^{1}$, a Bell inequality can be used to benchmark the ability to entangle and extract information from an entangled two-qubit system $^{2}$. Using the Clauser-Horne-Shimony-Holt (CHSH) variant $^{3}$ of the Bell test, this violation has been demonstrated with photons ${ }^{4,5}$, atoms ${ }^{6,7}$, solid-state spins ${ }^{8}$ and artificial atoms in superconducting circuits ${ }^{9,10}$. However, quantum computation necessitates the entanglement of large numbers of qubits. To perform tasks such as quantum error correction, a physical implementation must be capable of high-fidelity multi-qubit entanglement, as well as the efficient detection multi-qubit observables. For these larger, more distinguishable states, creating and preserving entanglement becomes increasingly difficult due to the rapid onset of decoherence ${ }^{11}$. Alternative encoding schemes that use coherent state superpositions, known as cat states ${ }^{12}$, take advantage of a cavity resonators much larger Hilbert space, as compared with that of a two-level system. This architecture allows redundant qubit encodings that can simplify the operations needed to initialize, manipulate and measure the encoded information ${ }^{13-15}$. For such a system to be viable as a quantum computing platform, efficient measurement of such encoded qubit observables must be possible. Using a circuit quantum electrodynamics architecture ${ }^{16}$, we show efficient, highfidelity measurements of an encoded cat state qubit and demonstrate this technology by detecting a violation of the $\mathrm{CHSH}$ Bell inequality between the encoded cat state qubit and a superconducting transmon qubit ${ }^{17}$. Furthermore, by the use of coherent states in this composite system, we can investigate the effects of decoherence by continuously varying the size of prepared entangled states ${ }^{18,19}$, something unachievable with discrete systems. These techniques provide an important set of analytical tools for quantum systems composed of entangled qubits and resonators ${ }^{14,19-23}$, and demonstrate that one can exploit coherent state superpositions in resonators without sacrificing measurement efficiency.

A resonator state can be completely described by direct measurements in the continuous variable basis with the cavity state Wigner function ${ }^{24}$. We extend this concept to express an entangled qubit-cavity state in what we call the joint Wigner representation. We construct this representation by performing a sequence of two quantum non-demolition measurements (Fig. 1), where a qubit state measurement is correlated with a subsequent cavity state measurement. However, complete cavity state tomography need not be required, and in fact many fewer measurements could be used to characterize a state when operating in a smaller, encoded subspace. By choosing an encoding scheme where states of a quantum bit are mapped onto a superposition of coherent states $|\beta\rangle$ and $|-\beta\rangle$, we can condense the joint Wigner representation down to just 16 correlations, equivalent to a two-qubit measurement set. Using direct fidelity estimation (DFE) ${ }^{25,26}$ and CHSH Bell witnesses ${ }^{27,28}$ within this logical basis, we investigate this systems susceptibility to decoherence by continuously increasing the cat state amplitude $\beta$. We measure a range in which correlations surpass the Bell inequality threshold and observe its reduction due to decoherence, benchmarking the efficiency of our encoding and detection schemes with cat state qubits.

\section{Results}

Creating the Bell-cat state. This experiment utilizes a circuit quantum electrodynamics architecture ${ }^{16,17}$ consisting of two waveguide cavities coupled to a single transmon qubit ${ }^{22,29}$.
One long-lived cavity (relaxation time $\tau_{\mathrm{s}}=55 \mu \mathrm{ms}$ ) is used for quantum information storage, while the other cavity, with fast field decay (relaxation time $\tau_{\mathrm{r}}=30 \mathrm{~ns}$ ), is used to realize repeated measurements. A transmon qubit (relaxation and decoherence times $T_{1}, T_{2} \approx 10 \mu \mathrm{s}$ ) is coupled to both cavity modes and mediates entanglement and measurement of the storage cavity state. All modes have transition frequencies between $5-8 \mathrm{GHz}$ and are off-resonantly coupled. The storage cavity and qubit mode are well described by the dispersive Hamiltonian:

$$
H / \hbar=\omega_{\mathrm{s}} a^{\dagger} a+\left(\omega_{\mathrm{q}}-\chi a^{\dagger} a\right)|e\rangle\langle e|
$$

where $a$ is the storage cavity ladder operator, $|e\rangle\langle e|$ is the excited state qubit projector, $\omega_{\mathrm{s}}$ and $\omega_{\mathrm{q}}$ are the storage cavity and qubit transition frequencies, and $\chi$ is the dispersive interaction strength between the two modes $(1.4 \mathrm{MHz})$. This interaction creates a shift in the transition frequency of one mode dependent on the other's excitation number, resulting in qubit-cavity entanglement ${ }^{30}$. As described in Fig. 1, the system is first prepared in a product state $|\psi\rangle=\frac{1}{\sqrt{2}}(|g\rangle+|e\rangle) \otimes|\beta\rangle$, where $|g\rangle$ and $|e\rangle$ are the ground and excited states of the qubit, and $|\beta\rangle$ is a coherent state of the cavity mode. Under the dispersive interaction, we allow the system to evolve for a time $t=\frac{\pi}{\chi}$, creating the entangled state:

$$
\left|\psi_{\mathrm{B}}\right\rangle=\frac{1}{\sqrt{2}}(|g, \beta\rangle+|e,-\beta\rangle),
$$

which we call a Bell-cat state $22,29,30$, mirroring the form of a twoqubit Bell state (for example, $|\psi\rangle=\frac{1}{\sqrt{2}}(|g g\rangle+|e e\rangle)$ ).

Correlating sequential high-fidelity measurements of the qubit and cavity allows state tomography of this composite system. We use a Josephson bifurcation amplifier ${ }^{31}$ in a double-pumped configuration in combination ${ }^{32,33}$ with a dispersive readout to perform repeated quantum non-demolition measurements with qubit detection fidelity of $98.0 \%$ at a minimum of 800 ns between measurements. With this sequence of two measurements, we characterize the efficacy of our entangling scheme and efficiency of measuring qubit-cavity observables with joint Wigner tomography, DFE and a CHSH inequality. The results of these tests illustrate our ability to recast the state encoded in the cavity as one that has a small, simple set of observables that directly mirrors that of the physical qubit.

Joint Wigner tomography. The first measurement detects the qubit along one of its basis vectors $\{X, Y, Z\}$. This value is recorded and the qubit is reset to $|g\rangle$ using real-time feedback. The displaced photon number parity observable $P_{\alpha}$ of the cavity is subsequently mapped onto the qubit using Ramsey interferometry ${ }^{24}$ before a second qubit state detection. The cavity observable $P_{\alpha}=D_{\alpha} P D_{\alpha}^{\dagger}$, where $D_{\alpha}$ is the displacement operator and $P$ the photon number parity operator, is detected with $95.5 \%$ fidelity. The Wigner function $W(\alpha)=\frac{2}{\pi}\left\langle P_{\alpha}\right\rangle$ is constructed from an ensemble of such measurements with different displacement amplitudes $\alpha$. The correlations between the qubit and cavity states make up what we refer to as the joint Wigner functions:

$$
W_{i}(\alpha)=\frac{2}{\pi}\left\langle\sigma_{i} P_{\alpha}\right\rangle,
$$

where $\sigma_{i}$ is an observable in the qubit Pauli set $\{I, X, Y, Z\}$. These four distributions are a complete representation of the combined qubit-cavity quantum state (Fig. 2). While other representations exist for similar systems ${ }^{34-37}, W_{i}(\alpha)$ is directly measured with this detection scheme and does not require a density matrix reconstruction. By an overlap integral (Supplementary Note 4), we determine the fidelity to a target state $\mathcal{F}=\left\langle\psi_{\mathrm{B}}|\rho| \psi_{\mathrm{B}}\right\rangle$ $=\frac{\pi}{2} \sum_{i} \int W_{i}^{\mathrm{B}}(\alpha) W_{i}(\alpha) d^{2} \alpha$, where $W_{i}^{\mathrm{B}}(\alpha)$ are the joint Wigner functions of the ideal state, $\left|\psi_{\mathrm{B}}\right\rangle$ and $W_{i}(\alpha)$ are the measured joint Wigner functions (normalized), yielding a state fidelity $\mathcal{F}=(87 \pm 2) \%$ for a displacement amplitude $\beta=\sqrt{3}$. This 

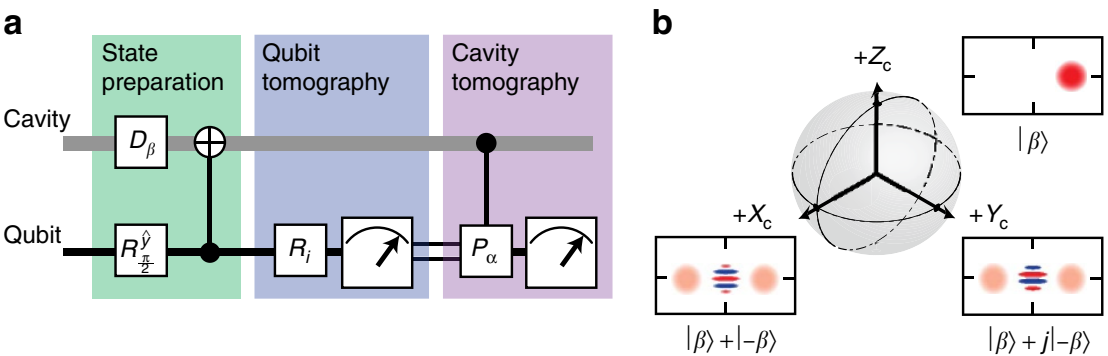

Figure 1 | Sequential detection for entanglement characterization. (a) A quantum circuit outlines the method to prepare and measure entanglement between a qubit and cavity state using sequential detection. State preparation is performed by first creating a product state $|\psi\rangle=\frac{1}{\sqrt{2}}(|g\rangle+|e\rangle) \otimes|\beta\rangle$ with a cavity displacement $D_{\beta}$ of amplitude $\beta$ and a qubit gate $R_{\frac{\pi}{2}}^{\hat{y}}$ corresponding to a $\frac{\pi}{2}$ rotation around the $\hat{y}$ axis. A conditional gate using the dispersive interaction produces the entangled state $\left|\psi_{\mathrm{B}}\right\rangle=\frac{1}{\sqrt{2}}(|g, \beta\rangle+|e,-\beta\rangle)$. Tomography is performed by measuring an observable of both the qubit and cavity with sequential quantum non-demolition measurements. A pre-rotation $R_{i}$ allows qubit detection along one of three basis vectors $X, Y$ and $Z$. The qubit is reset and a cavity observable $P_{\alpha}$ is mapped to the qubit for a subsequent measurement, where $P_{\alpha}=D_{\alpha} P D_{\alpha}^{\dagger}$ is the displaced photon number parity operator. Sequential detections are binary results compared shot-by-shot to determine qubit-cavity correlations. (b) The space spanned by the superposition of quasi-orthogonal coherent states $|\beta\rangle,|-\beta\rangle$ constitutes an encoded quantum bit in the cavity. While the cavity state can be represented by its Wigner function, this logical state is also described by a vector within its encoded Bloch sphere. Shown is the encoded qubit bloch sphere denoting the $+X_{c},+Y_{c}$ and $+Z_{c}$ encoded states; a diagram of the cavity Wigner function accompanies each of these three states. For well-separated coherent state superpositions, the entangled state $\left|\psi_{B}\right\rangle$ is then equivalent to a two-qubit Bell state.

a

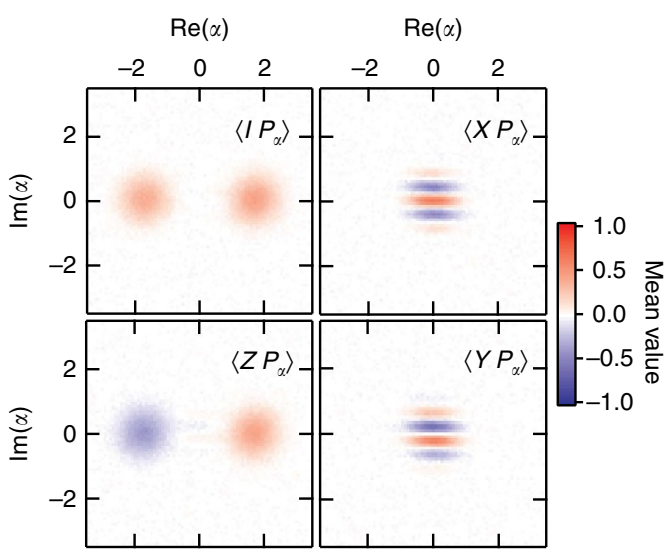

b $\mathrm{g} \quad \mathrm{e}$

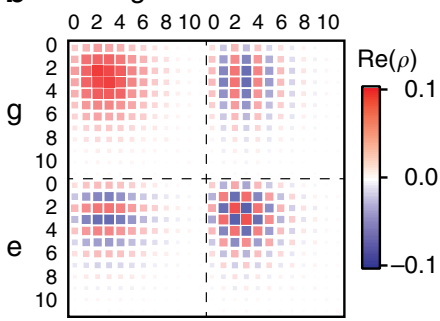

C

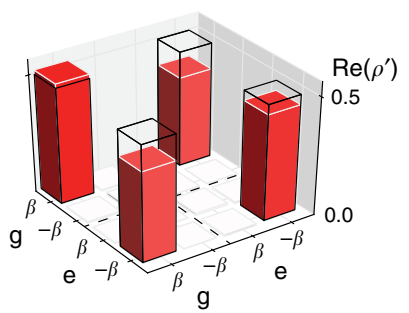

Figure 2 | Joint Wigner tomography of a Bell-cat state. (a) The set of joint Wigner functions $W_{i}(\alpha)=\frac{2}{\pi} \sigma_{i} P_{\alpha}$ represents the state of a qubit-cavity system with correlations between the qubit observables $\sigma_{i}=\{I, X, Y, Z\}$ and cavity observable $P_{\alpha}$ reported for a state $\left|\psi_{\mathrm{B}}\right\rangle$ and displacement amplitude $\beta=\sqrt{3}$. Shown are measurements comprised of four panels $I P_{\alpha,} X P_{\alpha,} Y P_{\alpha}$ and $Z P_{\alpha}$ of 6,500 correlations each between the qubit and cavity states. Interference fringes in $X P_{\alpha}$ and $Y P_{\alpha}$ reveal quantum coherence in the entangled state. (b) From the set of joint Wigner functions, we performed a density matrix reconstruction to show the combined qubit-cavity state $\rho$ in the Fock state basis. (c) Projecting $\rho$ onto the logical basis $\Phi=|\beta\rangle\langle\beta|+|-\beta\rangle\langle-\beta|$, produces the reduced, unnormalized density matrix $\rho^{\prime}=\Phi \rho \Phi^{\dagger}$ in the form of a traditional Bell state. The reduction in contrast of the off-diagonal components in $\rho^{\prime}$ is due to decoherence in the physical system during preparation and measurement.

amplitude was chosen to ensure orthogonality between logical states $|\langle\beta \mid-\beta\rangle|^{2}=6 \times 10^{-5} \ll 1$ with minimal tradeoff due to photon loss. Furthermore, the efficiency of our detection scheme can be quantified by the visibility of the unnormalized joint Wigner measurements $\mathcal{V}=\frac{2}{\pi} \int\left\langle I P_{\alpha}\right\rangle d^{2} \alpha=(85 \pm 1) \%$. Visibility $\mathcal{V}$ is primarily limited by measurement fidelity and qubit decoherence between detection events (Supplementary Note 5). The parameters $\mathcal{F}$ and $\mathcal{V}$ represent critical benchmarks for creating and retrieving information from entangled states.

Direct fidelity estimation. The number of measurement settings required to perform cavity state tomography can be resource intensive. Restricting to an encoded qubit subspace, only four values of the cavity Wigner function $W(\alpha)$ are required to reconstruct the state, known as a $\mathrm{DFE}^{25,26}$. For large cat states $|\langle\beta \mid-\beta\rangle|^{2} \ll 1$, the encoded state observables map to cavity observables as:

$$
\begin{array}{ll}
X_{\mathrm{c}}=P_{0} & I_{\mathrm{c}}=P_{\beta}+P_{-\beta} \\
Y_{\mathrm{c}}=P_{\frac{j \pi}{8 \beta}} & Z_{\mathrm{c}}=P_{\beta}-P_{-\beta},
\end{array}
$$

where $\left\{I_{c}, X_{c}, Y_{c}, Z_{c}\right\}$ form the Pauli set for the encoded qubit state in the cavity (Supplementary Note 8). Cuts in the joint Wigner function (Fig. 3) show these observables and their correlations to the qubit as a function of cat state size. As the superposition state is made larger, interference fringe oscillations increase, while fringe amplitude decreases due to photon loss. For a state $\left|\psi_{\mathrm{B}}\right\rangle$ with $|\beta|=\sqrt{3}$, we estimate a direct fidelity $\mathcal{F}_{\mathrm{DFE}}=\frac{1}{4}\left(\left\langle I I_{\mathrm{c}}\right\rangle+\right.$ 
$\left.\left\langle X X_{\mathrm{c}}\right\rangle-\left\langle Y Y_{\mathrm{c}}\right\rangle+\left\langle Z Z_{\mathrm{c}}\right\rangle\right)=(72 \pm 2) \%$ putting a fidelity bound on the target state with no corrections for visibility. This estimate is related to the benchmarks reported above $\mathcal{F}_{\mathrm{DFE}} \approx \mathcal{V} \times \mathcal{F}$ and far surpasses the $50 \%$ threshold for a classically correlated state. This indicates both high-fidelity state preparation and measurement, and demonstrates that strong correlations are directly detectable using joint Wigner tomography.

Bell inequality measurements. To place a stricter bound on observed entanglement, we perform a Bell test on the measured state. Although proposed to investigate local hidden variable theory, the Bell test here serves to benchmark the performance of a system that creates and measures entangled states ${ }^{8,9,10}$. Bell tests using homodyne measurements have been proposed ${ }^{38,39}$; however, here we choose the $\mathrm{CHSH}$ Bell test which states that the sum of four classical correlations will be bounded such that:

$$
-2 \leq \mathcal{O}=\left\langle A A_{\mathrm{c}}\right\rangle+\left\langle A B_{\mathrm{c}}\right\rangle-\left\langle B A_{\mathrm{c}}\right\rangle+\left\langle B B_{\mathrm{c}}\right\rangle \leq 2
$$

where, in this experiment, $A$ and $B$ are two qubit observables and $A_{\mathrm{c}}$ and $B_{\mathrm{c}}$ are two cavity observables. We perform two Bell tests (Fig. 4) with correlations taken shot by shot with no post selection
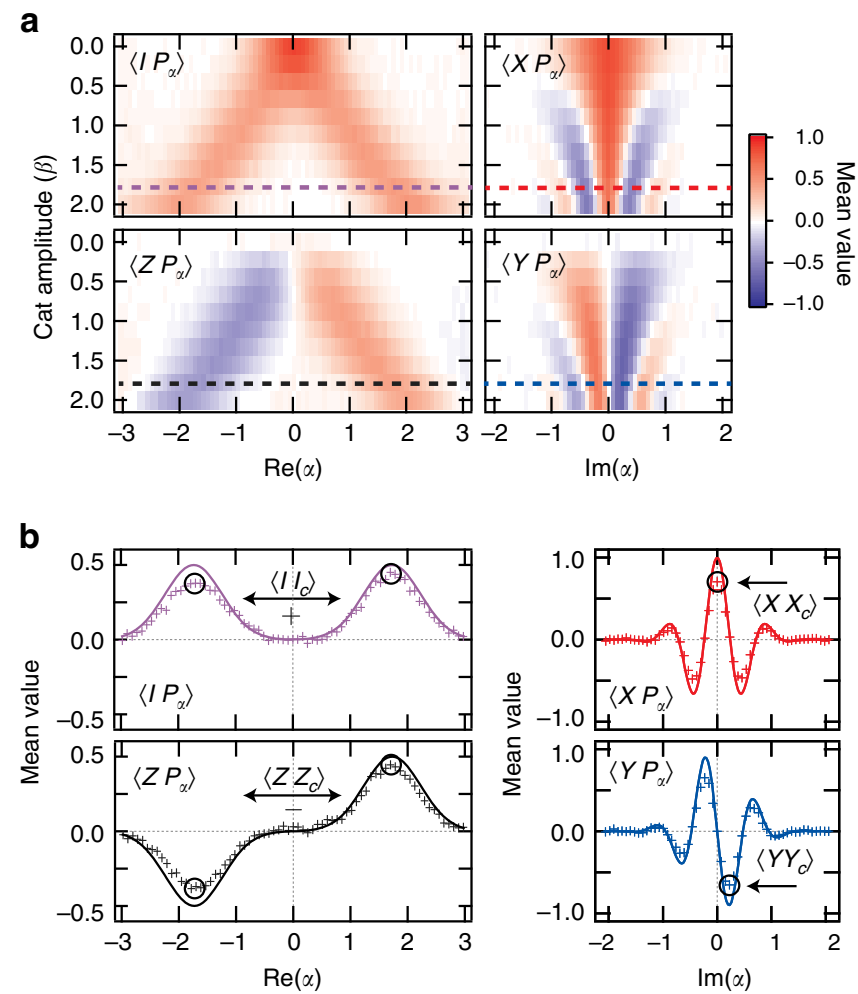

Figure 3 | Qubit-cavity correlations. (a) Correlations are measured for entangled states $\left|\psi_{B}\right\rangle$ with cat state amplitudes ranging from $\beta=0$ to 2 . Cuts in joint Wigner functions $I P_{\alpha}$ and $Z P_{\alpha}$ at $\operatorname{Im}(\alpha)=0$ show the increasing separation of the coherent state superpositions. Cuts in the joint Wigner functions $X P_{\alpha}$ and $Y P_{\alpha}$ at $\operatorname{Re}(\alpha)=0$ reveal the interference fringe oscillations dependence on cat state size, which increase in frequency with increasing cat state amplitude. (b) By viewing just single cuts at $\beta=\sqrt{3}$, we see single-shot correlations (crosses) as compared with what is expected from an ideal system with perfect preparation and measurement (solid line). From the cuts in $\mathbf{b}$ we see the individual measurement settings used to determine joint encoded observables $\left\{I_{c}, X X_{c}, Y Y_{c}, Z Z_{c}\right\}$. While $X X_{c}$ and $Y Y_{c}$ can be determined from a single measurement setting, $I I_{c}$ and $Z Z_{c}$ are determined from the sum and difference of two different settings. From these four correlations, we immediately find a fidelity to an entangled state $\mathcal{F}_{\text {DFE }}=\frac{1}{4}\left(\left\langle I_{c}\right\rangle+\left\langle X X_{c}\right\rangle-\left\langle Y Y_{c}\right\rangle+\left\langle Z Z_{c}\right\rangle\right)=(72 \pm 2) \%$. or compensation for detector inefficiencies. In the first, we take observables $X(\theta)=X \cos (\theta / 2)+Z \sin (\theta / 2), \quad Z(\theta)=Z \cos (\theta / 2)-$ $X \sin (\theta / 2), X_{c}, Z_{c}$ and sweep both qubit detector angle $\theta$ (Supplementary Note 11) and cat state amplitude $\beta$. We observe a Bell signal with a maximal value $\mathcal{O}_{1}=2.30 \pm 0.04$ at $\theta=-\frac{\pi}{4}$ for $\beta=1$. We witness a Bell signal surpassing bounded values up to cat states of size $|\beta-(-\beta)|^{2}=16$ photons ${ }^{19,29}$.

Measurements along $Z_{\mathrm{c}}$ require assumptions on the symmetry of the prepared state (Supplementary Note 8 ); we can instead employ an alternative Bell test. Using a scheme similar to ref. 28, and choosing observables $X, Y, X_{\mathrm{c}}(\alpha)=D_{j \alpha} X_{\mathrm{c}} D_{j \alpha}^{\dagger}, Y_{\mathrm{c}}(\alpha)=D_{j \alpha} Y_{\mathrm{c}} D_{j \alpha}^{\dagger}$, where $\alpha$ is a displacement amplitude corresponding to a rotation of the encoded cavity state detector (Supplementary Note 11), we observe a maximal value $\mathcal{O}_{2}=2.14 \pm 0.03$ for $\beta=1$. A lower Bell signal is observed in the second test due to its greater sensitivity to photon loss, yet in both tests two regimes are evident. For small cat state amplitudes, the initial Bell signal is limited by the nonorthogonality of the coherent state superpositions (Supplementary Note 7), while for large displacements the system's sensitivity to photon loss results in a reduction of the Bell signal. Larger, more distinguishable states quickly devolve into a classical mixture due to the onset of decoherence, corresponding to the resolution of Schrödinger's thought experiment. However, for intermediate cat state sizes, we observe Bell signals surpassing classical predictions larger than statistical uncertainties in both tests.

\section{Discussion}

In this letter, we have demonstrated the efficient detection of an artificial atom and a cat state in a cavity mode. We determine the entangled state using sequential detection with high-fidelity state measurement and real-time feedback on the quantum state. We benchmark the capabilities of this detection scheme with DFE and Bell test witnesses, which both reveal non-classical correlations of our system. Besides characterizing the high degree of entanglement in our Bell-cat, the tests detailed above also demonstrate that simple encoding techniques allow for the efficient extraction of information from states stored in a cavity, illustrating the viability of measuring redundantly encoded states in multi-level systems ${ }^{13,14}$. Furthermore, this implementation provides a resource for quantum state tomography and quantum process tomography of continuous variable systems and creates a platform for measurement-based quantum computation and quantum error correction using superconducting cavity resonators ${ }^{15}$. Finally, these features can extend to multi-cavity system ${ }^{27}$, which will require entanglement detection between continuous variable degrees of freedom and entanglement distribution of complex oscillator states.

\section{Methods}

Measurement set-up. Experiments are performed in a cryogen-free dilution refrigerator at a base temperature of $\sim 10 \mathrm{mK}$. Our output signal amplification chain consists of two stages. A Josephson bifurcation amplifier ${ }^{31}$ operating in a double-pumping configuration ${ }^{32,33}$ serves as the first stage, which is followed by a high electron mobility transistor amplifier.

Fabrication techniques of the transmon qubit and the design of storage and readout resonators follow the methods described in ref. 29. The refrigerator wiring (Fig. 5), including the filters and attenuators used, are similar to that of ref. 22, but with the addition of a feedback system, the details of which are discussed in a following section.

Qubit-cavity parameters. The two-cavity, single-qubit system is well described by the approximate dispersive Hamiltonian:

$$
\begin{aligned}
H / \hbar & =\omega_{\mathrm{s}} a_{\mathrm{s}}^{\dagger} a_{\mathrm{s}}+\omega_{\mathrm{r}} a_{\mathrm{r}}^{\dagger} a_{\mathrm{r}}+\omega_{\mathrm{q}} b^{\dagger} b \\
& -\frac{K_{\mathrm{s}}}{2} a_{\mathrm{s}}^{\dagger^{2}} a_{\mathrm{s}}^{2}-\frac{K_{\mathrm{r}}}{2} a_{\mathrm{r}}^{\dagger^{2}} a_{\mathrm{r}}^{2}-\frac{K_{\mathrm{q}}}{2} b^{\dagger^{2}} b^{2} \\
& -\chi_{\mathrm{qs}} a_{\mathrm{s}}^{\dagger} a_{\mathrm{s}} b^{\dagger} b-\chi_{\mathrm{qr}} a_{\mathrm{r}}^{\dagger} a_{r} b^{\dagger} b-\chi_{\mathrm{rs}} a_{\mathrm{s}}^{\dagger} a_{\mathrm{s}} a_{\mathrm{r}}^{\dagger} a_{\mathrm{r}}
\end{aligned}
$$



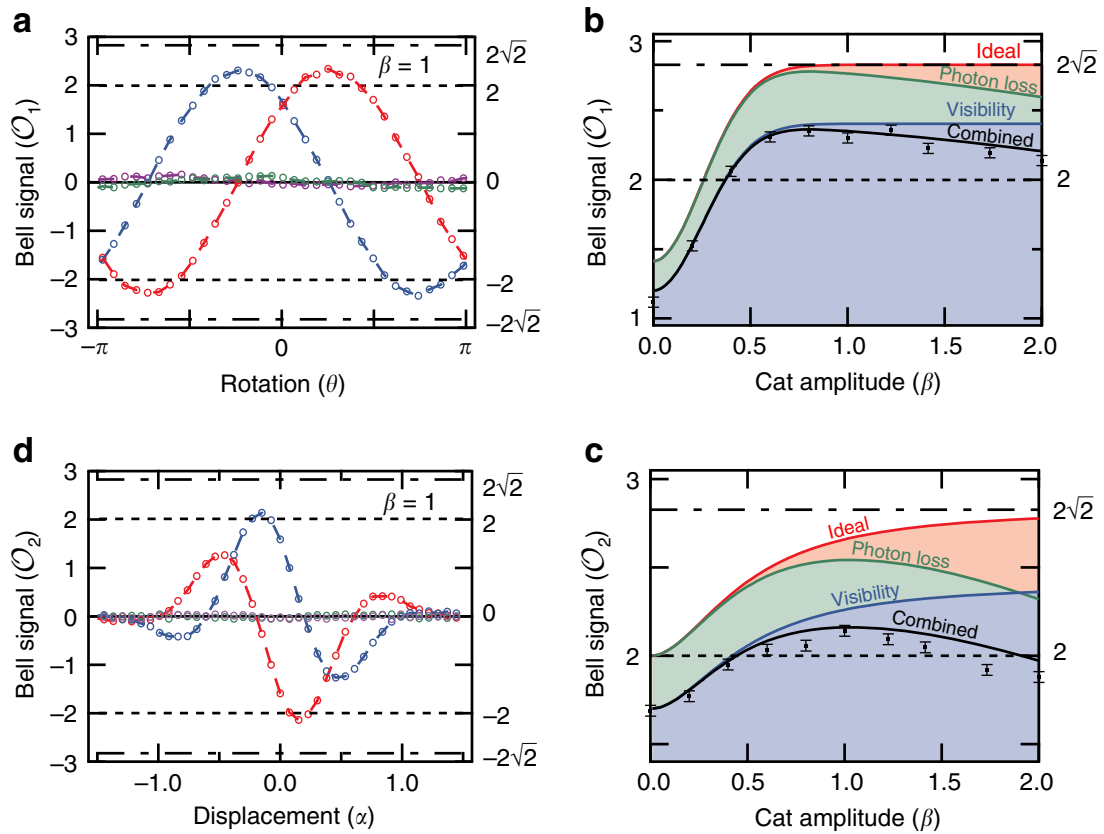

Figure 4 | Bell tests with a cat state. $\mathrm{A} C \mathrm{CHSH}$ Bell test between a qubit and cavity is the sum of four correlations $\mathcal{O}=\left\langle A A_{c}\right\rangle+\left\langle A B_{c}\right\rangle-\left\langle B A_{c}\right\rangle+\left\langle B B_{c}\right\rangle$, where $A$ and $B$ are observables of the qubit, and $A_{c}$ and $B_{c}$ are observables of the cavity. (a) We use correlations between qubit state observables $X(\theta)=X$ $\cos (\theta / 2)+Z \sin (\theta / 2)$ and $Z(\theta)=Z \cos (\theta / 2)-X \sin (\theta / 2)$ and encoded state observables $X_{c}$ and $Z_{c}$ to perform a CHSH Bell test as a function of qubit detector angle $\theta$. Shown in a are four traces that are the result of every possible combination of $X, Z, X_{c}$ and $Z_{c}$. A maximum Bell signal is found at $\theta=-\frac{\pi}{4}$. (b) We report this maximum Bell signal for different cat state amplitudes $\beta$. Plotted points (black) are the average Bell signal for a given amplitude and show the dependence of the entangled state with photon loss and detector visibility. Error bars denote the s.d. of the average signal due to random error as a consequence of a limited sample size $(N=4,000)$. Solid lines describe the predicted trends given the measured cavity decay rate and detection visibility. While the ideal behaviour (red) for an entangled state approaches $\mathcal{O}=2 \sqrt{2}$, photon loss (green), detector visibility (blue) and their combined effects (black) will ultimately limit the maximum Bell signal achieved. (c,d) Furthermore, we realize a second Bell test using qubit observables $X$ and $Y$, and cavity state observables $X_{c}(\alpha)=D_{j \alpha} X_{c} D_{j \alpha}^{\dagger}$ and $Y_{c}(\alpha)=D_{j \alpha} Y_{c} D_{j \alpha}^{\dagger}$, where $\alpha$ corresponds to a tomography displacement amplitude serving as a rotation of the effective cavity detector angle. There is a mismatch in the maxima obtained in the two different Bell tests due to increased susceptibility to photon loss in the second test. (c,d) Both, however, show a violation at least four s.d.'s beyond the classical limit defined by the CHSH Bell inequality.

Where $\omega_{\mathrm{s}}, \omega_{\mathrm{r}}$ and $\omega_{\mathrm{q}}$ are the storage, readout and qubit transition frequencies, $a_{\mathrm{s}}$, $a_{\mathrm{r}}$ and $b$ are the associated ladder operators, and $K$ and $\chi$ are the modal anharmonicities and dispersive shifts, respectively. Supplementary Table 1 details the Hamiltonian parameters of our system. The resonant frequency of the readout resonator $\omega_{\mathrm{r}} / 2 \pi$ is determined by transmission spectroscopy. The qubit frequency $\omega_{s} / 2 \pi$ and storage cavity frequencies $\omega_{\mathrm{q}} / 2 \pi$ are found using two-tone spectroscopy.

Qubit anharmonicity $K_{\mathrm{q}}$ is measured using two-tone spectroscopy to observe the $0-2$ two-photon transition ${ }^{17}$. Storage cavity anharmonicity $K_{\mathrm{s}}$ is determined by displacing the cavity with a coherent state and observing its time evolution with Wigner tomography. The resulting dynamics are characterized by state reconstruction and $K_{\mathrm{s}}$ is observed by the state's quadratic dependence of phase on photon number. Finally, we predict the readout cavity anharmonicity $K_{\mathrm{r}}$ using its approximate dependence on the measured values of $K_{\mathrm{q}}$ and the qubit-readout dispersive shift $\chi_{\mathrm{gr}}$ (ref. 40).

The dispersive shift between the qubit and the readout resonator $\chi_{\mathrm{qr}}$ is found by taking the difference in frequency between the readout resonance when the qubit is in the ground and excited state. The dispersive shift between the qubit and the storage resonator $\chi_{\mathrm{qs}}$ is found using two methods: photon number-dependent qubit spectroscopy ${ }^{41}$, and observing qubit state revival using Ramsey interferometry ${ }^{29}$. Finally, $\chi_{\mathrm{rs}}$ is predicted using its approximate relationship between $K_{\mathrm{s}}$ and $K_{\mathrm{r}}$ (ref. 40).

Lifetimes and thermal populations. The lifetime of the storage cavity is determined by displacing to a coherent state, waiting a variable length of time, and then applying a qubit rotation conditioned on zero photons in the storage cavity. This allows a measurement of the time-dependent overlap of the cavity state with its ground state $|0\rangle$ dependent on time. The lifetime of the readout cavity is found from its line width. The thermal population of the qubit is determined from a histogram of one million single-shot measurements of the qubit thermal state, where the signal-to-noise ratio provided by the Josephson bifurcation amplifier allows discrimination between $|g\rangle$ and all states not $|g\rangle$. The thermal population of the storage cavity is found by taking the difference between parity measurements of the thermal and vacuum states of the cavity. A vacuum state is prepared by first performing two parity measurements on the thermal state and then post-selecting such that all results give even parity, projecting the thermal state onto $|0\rangle$. Finally, the known thermal population of the readout cavity is bounded by the dephasing rate $\Gamma_{\phi}$ of the qubit: $\Gamma_{\phi}=\bar{n}_{\text {th }} \kappa$, where $\bar{n}_{\text {th }}$ is the readout cavity's thermal occupation and $\kappa$ is the readout single-photon decay rate ${ }^{42}$. Coherence properties are summarized in Supplementary Table 2.

Measurement fidelities. We define single-shot measurement fidelity as $F_{\mathrm{q}}=\frac{P(g \mid g)+P(e \mid e)}{2}$, where $P(g \mid g)$ and $P(e \mid e)$ are the probabilities to get $|g\rangle(|e\rangle)$, knowing that we start with $|g\rangle(|e\rangle)$. The state $|g\rangle$ is prepared through purification of the qubit thermal state with real-time feedback (see the following section) Given a preparation of $|g\rangle$, we have a $98.5 \%$ chance of measuring $|g\rangle$ again $(P(g \mid g)=0.985)$. Likewise, we find $P(e \mid e)=0.975$ by preparing $|g\rangle$ and rotating the state to $|e\rangle$. This gives a single-shot measurement fidelity of $F_{\mathrm{q}}=98 \%$. We find our cavity parity measurement fidelity by purifying the storage cavity thermal state into $|0\rangle$ then performing one of two kinds of parity measurement (Supplementary Figs 7 and 8 ; Supplementary Table 3 ). We report a parity measurement fidelity for $n=0$ photons as $F_{\mathrm{c}}=\frac{P\left(g \mid E_{1}\right)+P\left(e \mid E_{2}\right)}{2}=95.5 \%$, where $P\left(g \mid E_{1}\right)$ and $\left(P\left(e \mid E_{2}\right)\right)$ are the probabilities to measure $|g\rangle(|e\rangle)$, given that the parity is even for each of the two measurement settings. We expect $F_{\mathrm{c}}$ to decrease with increasing numbers of photons in the cavity due to single-photon loss during the measurement sequence

Directly from these readout fidelities, the estimated visibility ${ }^{43}$ for correlated observables $\mathcal{V}_{\text {est }}=\left(2 F_{\mathrm{q}}-1\right)\left(2 F_{\mathrm{c}}-1\right)=87 \%$. This allows us to predict the maximum Bell violation possible given only measurement inefficiencies $\mathcal{O}_{\max }=2 \sqrt{2} \mathcal{V}_{\text {est }}=2.47$. In practice, $\mathcal{V}$ is directly related to the contrast of the joint Wigner function (Supplementary Note 5), which we measure to be $85 \%$. This discrepancy is due to qubit decoherence, which is studied further in Supplementary Note 2, and puts a more conservative estimate for the maximum Bell violation achievable: $\mathcal{O}_{\max }=2 \sqrt{2} \mathrm{~V}=2.40$.

I/O control parameters. As shown in Fig. 5, we employ a field-programmable gate array (FPGA) to implement an active feedback scheme. We use an X6-1000M board from Innovative Integration that contains two $1 \mathrm{GS} / \mathrm{s}$ analogue-to-digital converters, two $1 \mathrm{GS} / \mathrm{s}$ digital-to-analogue converter channels and digital 


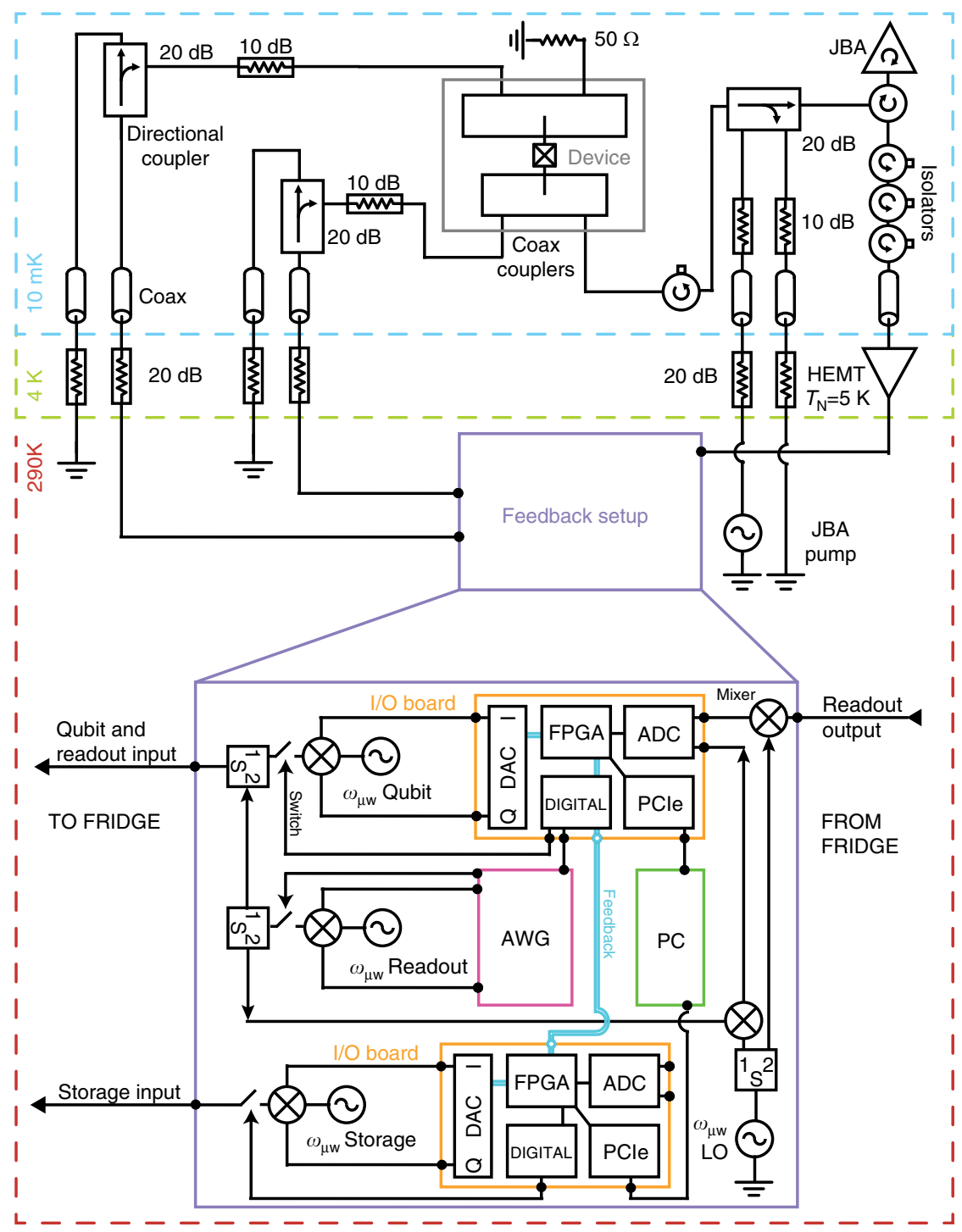

Figure 5 | Experiment schematic. Schematic of the experiment shows a two-cavity one-qubit device identical to that used in ref. 22 , however with the addition of a feedback set-up for active qubit reset. The feedback set-up uses two input-output $(\mathrm{I} / \mathrm{O})$ boards for qubit and storage resonator control and one arbitrary waveform generator (AWG) for readout resonator control. All have a dedicated microwave generator and mixer for amplitude and phase modulation. Each I/O board has five main components: (1) a digital-to-analogue converter (DAC) for pulse generation; (2) digital outputs serving as marker channels; (3) an analogue-to-digital converter (ADC) that samples input signals; (4) an FPGA that demodulates the signals from the ADC and based on predefined thresholds determines the measured qubit state, $|g\rangle$ or $|e\rangle$ to generate pulses; and (5) a PCle connection that transfers FPGA data to a computer (PC) for analysis. In this set-up, the top I/O board serves as the master, which accepts the readout signal, returns qubit state information, and using digital output signals, triggers the AWG and the second I/O card given a particular qubit measurement result.

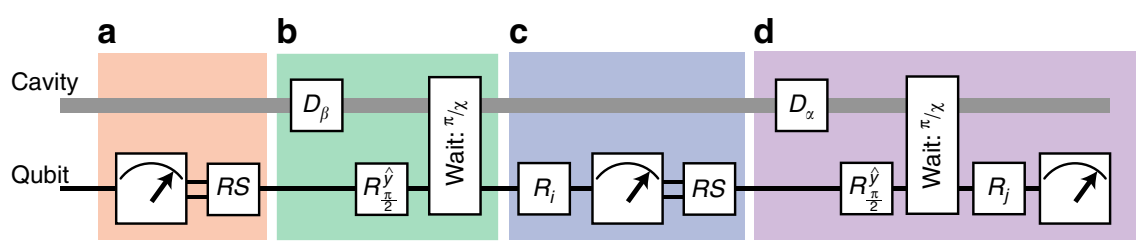

Figure 6 | Full quantum circuit diagram. Each experiment is split into four components. (a) First, the system is initialized. The qubit state is measured and a qubit pulse $R S=\left\{R_{\hat{y}}^{\pi}\right.$ or 1$\}$ is applied to reset the qubit to $|g\rangle$. (b) Second, the entangled state is created with a cavity displacement $D_{\beta}$ and a qubit rotation $R_{\hat{y}}^{\pi / 2}$ followed by a $\frac{\pi}{\chi}$ waiting time to produce the entangled state $\left|\psi_{B}\right\rangle=\frac{1}{\sqrt{2}}(|g, \beta\rangle+|e,-\beta\rangle)$. (c) Following preparation, a qubit state detection is performed with a pre-rotation $R_{i}$ (Supplementary Table 3), a measurement, and a qubit reset RS. Finally, we perform a cavity state measurement using Ramsey interferometry, where $R_{j}= \pm R_{\hat{y}}^{\pi / 2}$ combined with an initial pre-displacement $D_{\alpha}$. This maps $P_{\alpha}$ to the qubit state, which is readout with a subsequent qubit measurement. Correlations are reported as the product of detection events between measurements in $\mathbf{c}$ and $\mathbf{d}$. 

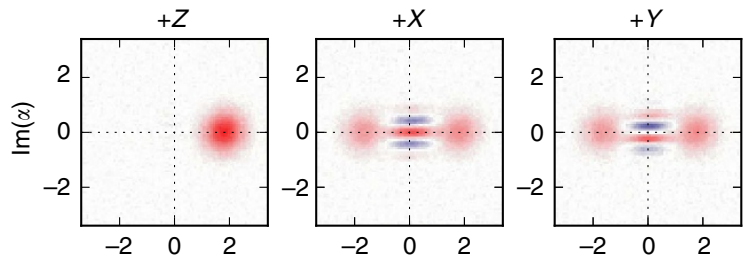

$\left\langle P_{\alpha}\right\rangle$
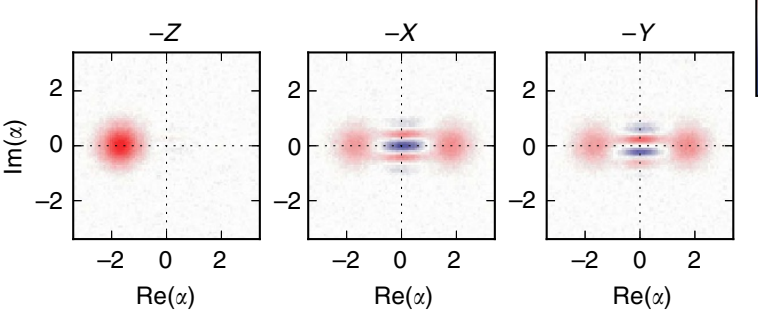

\section{0}

0.5

0.0

$-0.5$

$-1.0$

Figure 7 | Qubit measurement back action of a Bell-cat state. Shown are the resulting projections of the cavity state when preparing $\left|\psi_{B}\right\rangle$ and measuring the qubit along one of its three axes $M q \in\{X, Y, Z\}$. While a measurement along $Z$ results in a projected coherent state with opposite phases $| \pm \beta\rangle$, measuring along the $X$ and $Y$ axes results in a projected cat state each with different inference fringe phases. Combining these measurements with the probability to obtain each result allows us to construct the state of the entire system and is used to create the joint Wigner function representation in Fig. 2.

inputs/outputs all controlled by a Xilinx VIRTEX-6 FPGA loaded with custom logic. We synchronize two such boards in a master/slave configuration to have IQ control of both the qubit/storage cavity. IQ control over the readout cavity is performed with a Tektronix AWG, which is triggered by the master board. The readout and reference signals are routed to the analogue-to-digital converters on the master board, whereafter the FPGA demodulates the signal and decides whether the qubit is in $|g\rangle$ or $|e\rangle$. The feedback latency of the FPGA logic (last in, first out LIFO) is $320 \mathrm{~ns}$. Additional delay for active feedback includes cable delay $(\sim 100 \mathrm{~ns})$ and readout pulse length with resonator decay time (320 ns). Thus, in total, the qubit waits $\tau_{\text {wait }} \sim 740$ ns between the time at which photons first enter the readout resonator and the time at which the feedback pulse resets the qubit.

Implementations of feedback. Feedback is used three times during a single iteration of the experiment. Before the state preparation (Fig. 6), we purify the qubit state to $|g\rangle$ by measuring the qubit and applying a rotation $R_{\tilde{y}}^{\pi}$ if measured in $|e\rangle$. We succeed in preparing $|g\rangle$ with a probability of $99 \%$. Second, when performing qubit tomography we reset the qubit to $|g\rangle$ if it is measured to be in $|e\rangle$. Since we must wait $\tau_{\text {wait }}$ before feedback can be applied, the cavity state will acquire an additional phase $\chi_{\mathrm{qs}} \tau_{\text {wait }}$ if the qubit is in $|e\rangle$. In this case, in addition to resetting the qubit, the FPGA applies an equivalent phase shift on the subsequent Wigner tomography pulse. This feedback implementation does not close the 'locality' loophole for a $\mathrm{CHSH}$ Bell test and therefore cannot be used to test local realism.

Quantum measurement back action. The sequential measurement protocol allows us to observe the result of quantum measurement back action of the qubit on the cavity state. We prepare the system in a Bell-cat state as in equation (2) and measure along one of the three qubit axes $M_{\mathrm{q}} \in\{X, Y, Z\}$. For each measurement, we observe one of two possible outcomes of the projected cavity state $\left|\psi_{\text {cav }}\right\rangle$ :

$$
\begin{array}{ccc}
M_{\mathrm{q}} & \text { result 1 } & \text { result 2 } \\
X & \mathcal{N}(|\beta\rangle+|\beta\rangle) & \mathcal{N}(|\beta\rangle-|\beta\rangle) \\
Y & \mathcal{N}(|\beta\rangle-j|\beta\rangle) & \mathcal{N}(|\beta\rangle+j|\beta\rangle) \\
Z & |\beta\rangle & |-\beta\rangle
\end{array}
$$

See Fig. 7 for each of these projective measurements on the Bell-cat state $\left|\psi_{\mathrm{B}}\right\rangle$. The method of using strong projective measurements for the create of cat states has been demonstrated in previous works ${ }^{19}$. A second example of quantum measurement back action using an entangled Fock state can be found in Supplementary Fig. 9.

\section{References}

1. Bell, J. S. Speakable and Unspeakable in Quantum Mechanics (Cambridge Univ. Press, 1987)

2. van Enk, S. J., Lütkenhaus, N. \& Kimble, H. J. Experimental procedures for entanglement verification. Phys. Rev. A 75, 052318 (2007).

3. Clauser, J. F., Horne, M. A., Shimony, A. \& Holt, R. A. Proposed experiment to test local hidden-variable theories. Phys. Rev. Lett. 23, 880-884 (1969).
4. Freedman, S. J. \& Clauser, J. F. Experimental test of local hidden-variable theories. Phys. Rev. Lett. 28, 938-941 (1972).

5. Aspect, A., Grangier, P. \& Roger, G. Experimental tests of realistic local theories via Bell's theorem. Phys. Rev. Lett. 47, 460-463 (1981).

6. Rowe, M. A. et al. Experimental violation of a Bell's inequality with efficient detection. Nature 409, 791-794 (2001).

7. Hofmann, J. et al. Heralded entanglement between widely separated atoms. Science 337, 72-75 (2012).

8. Pfaff, W. et al. Demonstration of entanglement-by-measurement of solid-state qubits. Nat. Phys. 9, 29-32 (2012).

9. Ansmann, M. et al. Violation of Bell's inequality in Josephson phase qubits. Nature 461, 504-506 (2009).

10. Chow, J. et al. Detecting highly entangled states with a joint qubit readout. Phys. Rev. A 81, 062325 (2010).

11. Lanyon, B. P. et al. Experimental violation of multipartite Bell inequalities with trapped ions. Phys. Rev. Lett. 112, 100403 (2014).

12. Haroche, S. \& Raimond, J.-M. Exploring the Quantum: Atoms, Cavities, and Photons (Oxford Univ. Press, 2006).

13. Gottesman, D., Kitaev, A. \& Preskill, J. Encoding a qubit in an oscillator. Phys. Rev. A 64, 012310 (2001).

14. Jeong, H. \& Kim, M. Efficient quantum computation using coherent states. Phys. Rev. A 65, 042305 (2002).

15. Leghtas, Z. et al. Hardware-efficient autonomous quantum memory protection. Phys. Rev. Lett. 111, 120501 (2013).

16. Wallraff, A. et al. Strong coupling of a single photon to a superconducting qubit using circuit quantum electrodynamics. Nature 431, 162-167 (2004).

17. Paik, H. et al. Observation of high coherence in Josephson junction qubits measured in a three-dimensional circuit QED architecture. Phys. Rev. Lett. 107, 240501 (2011).

18. Brune, M. et al. Observing the Progressive Decoherence of the "Meter" in a Quantum Measurement. Phys. Rev. Lett. 77, 4887-4890 (1996).

19. Deléglise, S. et al. Reconstruction of non-classical cavity field states with snapshots of their decoherence. Nature 455, 510-514 (2008).

20. Leibfried, D. et al. Experimental Determination of the Motional Quantum State of a Trapped Atom. Phys. Rev. Lett. 77, 4281-4285 (1996).

21. Hofheinz, M. et al. Synthesizing arbitrary quantum states in a superconducting resonator. Nature 459, 546-549 (2009).

22. Sun, L. et al. Tracking photon jumps with repeated quantum non-demolition parity measurements. Nature 511, 444-448 (2014).

23. Leghtas, Z. et al. Confining the state of light to a quantum manifold by engineered two-photon loss. Science 347, 853-857 (2015).

24. Lutterbach, L. G. \& Davidovich, L. Method for direct measurement of the Wigner function in cavity QED and ion traps. Phys. Rev. Lett. 78, 2547-2550 (1997).

25. da Silva, M., Landon-Cardinal, O. \& Poulin, D. Practical Characterization of Quantum Devices without Tomography. Phys. Rev. Lett. 107, 210404 (2011).

26. Flammia, S. T. \& Liu, Y.-K. Direct fidelity estimation from few pauli measurements. Phys. Rev. Lett. 106, 230501 (2011).

27. Milman, P. et al. A proposal to test Bell's inequalities with mesoscopic nonlocal states in cavity QED. Eur. Phys. J. D 32, 233-239 (2004).

28. Park, J., Saunders, M., Shin, Y.-i., An, K. \& Jeong, H. Bell-inequality tests with entanglement between an atom and a coherent state in a cavity. Phys. Rev. A 85, 022120 (2012).

29. Vlastakis, B. et al. Deterministically Encoding Quantum Information Using 100-Photon Schrodinger Cat States. Science 342, 607-610 (2013).

30. Brune, M., Haroche, S., Raimond, J., Davidovich, L. \& Zagury, N. Manipulation of photons in a cavity by dispersive atom-field coupling: Quantumnondemolition measurements and generation of "Schrödinger cat" states. Phys. Rev. A 45, 5193-5214 (1992).

31. Vijay, R., Devoret, M. H. \& Siddiqi, I. The Josephson bifurcation amplifier. Rev. Sci. Instrum. 80, 111101 (2009).

32. Kamal, A., Marblestone, A. \& Devoret, M. Signal-to-pump back action and self-oscillation in double-pump Josephson parametric amplifier. Phys. Rev. B 79, 184301 (2009).

33. Murch, K. W., Weber, S. J., Macklin, C. \& Siddiqi, I. Observing single quantum trajectories of a superconducting quantum bit. Nature 502, 211-214 (2013).

34. Eichler, C. et al. Observation of Entanglement Between Itinerant Microwave Photons and a Superconducting Qubit. Phys. Rev. Lett. 109, 240501 (2012).

35. Morin, O. et al. Remote creation of hybrid entanglement between particle-like and wave-like optical qubits. Nat. Photon. 8, 570-574 (2014).

36. Jeong, H. et al. Generation of hybrid entanglement of light. Nat. Photon. 8, 564-569 (2014).

37. LinPeng, X. Y. et al. Joint quantum state tomography of an entangled qubit-resonator hybrid. New. J. Phys. 15, 125027 (2013).

38. Leonhardt, U. \& Vaccaro, J. A. Bell correlations in phase space: application to quantum optics. J. Modern Optics 42, 939-943 (1995).

39. Gilchrist, A., Deuar, P. \& Reid, M. D. Contradiction of quantum mechanics with local hidden variables for quadrature phase amplitude measurements. Phys. Rev. Lett. 80, 3196 (1998). 
40. Nigg, S. E. et al. Black-box superconducting circuit quantization. Phys. Rev. Lett. 108, 240502 (2012).

41. Schuster, D. I. et al. Resolving photon number states in a superconducting circuit. Nature 445, 515-518 (2007).

42. Sears, A. P. et al. Photon shot noise dephasing in the strong-dispersive limit of circuit QED. Phys. Rev. B 86, 180504 (2012).

43. Kofman, A. G. \& Korotkov, A. N. Analysis of Bell inequality violation in superconducting phase qubits. Phys. Rev. B 77, 104502 (2008).

\section{Acknowledgements}

We thank R. Heeres, W. Pfaff and A. Narla for discussions. This research was supported by the National Science Foundation (NSF; PHY-1309996), the Multidisciplinary University Research Initiatives program through the Air Force Office of Scientific Research (FA9550-14-1-0052) and the US Army Research Office (W911NF-14-1-0011), Facilities use was supported by the Yale Institute for Nanoscience and Quantum Engineering and the NSF (MRSECDMR 1119826).

\section{Author contributions}

B.V. and A.P. performed the experiment and analysed the data. N.O. and Y.L. designed the feedback architecture. L.S. provided further experimental implementation support.

K.S. and M.H. provided the Josephson Parametric Converter technology under the supervision of M.H.D. Z.L., M.M. and L.J. provided theoretical support. J.B. and L.F. fabricated the transmon qubit. R.J.S. supervised the project. B.V., A.P., L.F. and R.J.S. wrote the manuscript with feedback from all authors.

\section{Additional information}

Supplementary Information accompanies this paper at http://www.nature.com/ naturecommunications

Competing financial interests: The authors declare no competing financial interests.

Reprints and permission information is available online at http://npg.nature.com/ reprintsandpermissions/

How to cite this article: Vlastakis, B. et al. Characterizing entanglement of an artificial atom and a cavity cat state with Bell's inequality. Nat. Commun. 6:8970 doi: $10.1038 /$ ncomms9970 (2015)

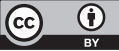

This work is licensed under a Creative Commons Attribution 4.0 International License. The images or other third party material in this article are included in the article's Creative Commons license, unless indicated otherwise in the credit line; if the material is not included under the Creative Commons license, users will need to obtain permission from the license holder to reproduce the material. To view a copy of this license, visit http://creativecommons.org/licenses/by/4.0/ 